\title{
Effectiveness of Neck Stretching Exercises on Neck Pain and Disability for Patients with Total Thyroidectomy
}

\section{Hagar Magdy AbdElhafiez ${ }^{1}$, Mostafa Mostafa Rezk², Sabah Said Mohamed ${ }^{3}$ and Marwa Mostafa $\mathrm{Ali}^{4}$}

(1) Assistant Lecturer at MTI University, Egypt (2) Professor of General Surgery - Faculty of MedicineBenha University- Egypt, (3) Assistant Professor of Medical Surgical Nursing- Faculty of NursingBenha University- Egypt and (4) Assistant Professor of Medical Surgical Nursing- Faculty of Nursing-Benha University - Egypt

\section{Abstract}

Background: Neck stretching exercises improves flexibility and neuromuscular coordination, reduces pain and muscle weakness, improves physical activity and encourages proper posture. Aim: This study aimed to evaluate the effectiveness of neck stretching exercises on neck pain and disability for patients with total thyroidectomy. Design: Quasi-experimental design was utilized to fulfill the aim of this study. Setting: This study was conducted in the surgical departments at Benha University Hospital and outpatient clinics. Subjects: Purposive sample of patients with total thyroidectomy (60 patients) divided into two groups (intervention group $n=30$ ) and (control group $n=30$ ) during the time of data collection (12months). Data collection tools: 1) Patients Structured Interviewing Questionnaire to evaluate patients total knowledge, 2) Neck disability index to evaluate neck disability level after total thyroidectomy and 3) Visual analogue scale for pain to evaluate neck pain level after total thyroidectomy. Results: The mean age of both studied groups (intervention\& control) was 39.20 \pm 7.45 $\& 40.51 \pm 7.70$, respectively. There were no significant statistical differences between them regarding total knowledge level during pre-operative period. However, it was significantly different during (postoperative $1^{\text {st }}$ week $\& 4^{\text {th }}$ week). There was highly statistically significant difference between both studied groups regarding neck pain and disability level in $1^{\text {st }}$ week postoperative period, while there were statistically significant differences between both studied groups regarding neck pain and disability level in $4^{\text {th }}$ week postoperative period. Conclusion: There were high significant improvement in the intervention group total knowledge level $1^{\text {st }}$ week and $4^{\text {th }}$ week post - operative following the exercises program implementation more than the control group, moreover the study group had a lower level of neck pain and disability than the control group $1^{\text {st }}$ week and $4^{\text {th }}$ week post-operative following the exercises program implementation. Recommendations: Replication of the study using a larger probability sample from different geographical areas to attain more generalizable results.

Keywords: Neck stretching exercises, Pain and disability of the neck, Total thyroidectomy

\section{Introduction}

Thyroid surgeries have tripled over the past three decades; between 118,000 to 166,000 patients in the United States undergo thyroidectomy each year (D'Orazi et al., 2019).

Thyroidectomy is the surgical removal of all or part of the thyroid gland. Thyroid surgery commonly falls into one of three categories; total thyroidectomy, with the aims of achieving complete macroscopic removal of the thyroid tissue; subtotal thyroidectomy, in which bilateral thyroid remnants are left and thyroid lobectomy which involves removal of half of the thyroid gland that has the nodule ( Abboud et al., 2012).

Although complications following total thyroidectomy are rare but their consequences 


\section{Thyroidectomy}

can be life-threatening, and includes; hypothyroidism, damage to or inadvertent removal of parathyroid glands causing hypoparathyroidism and hypocalcemia, hemorrhage, injury to the recurrent or superior laryngeal nerve, thyrotoxicosis, cervical hematomas and wound infection. Also the patients have postoperative occipital headaches, neck pain and discomfort, neck and shoulder movement problems, stiffness in the shoulders, and limitation of cervical spine range of motion as a result of the fixed position of neck during and after thyroidectomy surgery. These previous mentioned complains may be occurred for a long-time following surgery and may affect negatively on the patients' quality of life (Christou \& Mathonnet, 2013).

Neck discomfort symptoms have negative effects on patients' quality of life after a thyroidectomy. Neck movements should be recommended for patients post operatively as a routine care to avoid those negative experiences. Neck stretching exercises, including basic movements of the neck, are known to be the simplest and most effective exercises. Stretching exercises improves flexibility and neuromuscular coordination, reduces pain and muscle weakness; it also improves physical activity encourages proper posture (Jang et al., 2014).

Nurses play an important role in informing patients about the signs and symptoms of potential complications after total thyroidectomy, also should assess pain severity and educate patient neck stretching exercises should be practiced even the patients have less pain level or have the ability to move their head, neck and shoulders freely. In addition to, written and verbal information about the surgery and neck stretching exercises, postoperative instructions about medications, wound care, nutrition, daily living activities and follow-up should be given to the patients.
Also, nurse should encourage patients to move and stretch their shoulders and neck slowly and completely because those exercises helps in decreasing pain and muscle weakness, improve functionality and flexibility level of the patients (Hameed,2018).

\section{Significant of the study}

The prevalence of different types of thyroid disease varied between the reported studies in Arab world from 2014 till 2016 ranging from 6.18 to $47.34 \%$ prevalence of goiter reported by several studies conducted in Egypt (Al Shahrani, et al. 2016). Patients frequently experience discomfort symptoms in their neck such as neck pain, stress and pressure in the neck, shoulder stiffness, neck and shoulder movement difficulties. In addition, during the early postoperative period, patients were observed in the current study to not move their necks and to walk robotically, possibly to prevent exacerbating the neck pain and to protect their incisions, as was similarly reported by (Takamura, et al. 2005). According to Benha University Hospital records from (2017 to 2018); incidence of thyroidectomy operations approximately was "200" procedures were done through this period. (Statistical Administration and Medical Records, at Benha University Hospital, 2019). So this study is important to be implemented to help such group of patients increasing their knowledge and practice in an attempt to decrease the incidence of this complication.

\section{Aim of the study}

The aim of the current study was to evaluate the effectiveness of neck stretching exercises on neck pain and disability for patients with total thyroidectomy.

\section{Research Hypothesis}

The study hypothesized that:

- The intervention group will exhibit higher knowledge level after implementing the exercises program compared to control group. 
- The intervention group will exhibit lower level of neck pain and disability after implementing the exercises program than control group.

\section{Subjects and Methods}

\section{Study design: -}

Quasi-experimental design was utilized to fulfill the aim of this study

\section{Setting:-}

The study was conducted in the surgical departments at Benha University Hospital and outpatient clinics.

\section{Subjects:-}

Purposive sample of patients with total thyroidectomy (60 patients) divided in to two groups (intervention group $\mathrm{n}=30$ ) and (control group $n=30$ ) during the time of data collection (12months), older than 25 years old and agreed to participate in this study.

Tools of data collection:

The following tools were constructed, tested and piloted by the researcher to collect data, it was used as a follow:

Tool one: Patients Structured Interviewing Questionnaire: It was developed by the researcher in simple Arabic language based on the extensive review of relevant and recent literatures, with the aim to collect data pertinent to socio-demographic patient characteristics, medical data, and knowledge regarding thyroid gland, thyroidectomy, neck stretching exercises and it will be divided into three parts:

\section{1-Socio-demographicdatacharacteristics}

including questions about (Age, gender, marital status, level of education, occupation and residence.

2- Medical data including questions about current medical history (period from diagnosis of disease till the operation, causes of thyroidectomy, complains after surgery and past medical history (previous surgery, chronic diseases, past family history for thyroidectomy).
3-Patient's knowledge regarding thyroid gland, thyroidectomy, and neck stretching exercises: It was adapted from (Lewis et al., 2016) \& (Ignatavicius \& Workman, 2015) including (17) questions distributed into two parts:

A- Knowledge about hypothyroidism it concluded (9 questions).

B- Knowledge about (neck stretching exercises and it concluded (8 questions).

Scoring system: all knowledge variables were weighted according to the items included in each question of multiple choices the question response (yes) scored as"1" but the question response (no) and (don't know) scored as "0".

* According to statistical report it was distributed as:

$>60 \%$ and above considered as
satisfactory.
$>$ Below $60 \%$ considered as
unsatisfactory.

Tool two: Neck pain and disability index questionnaire (NDI): It was adapted from (Vernon \& Mior, 1991) to give information as to how neck pain after operation has affected the ability to manage everyday life. NDI consists of ten items; pain intensity, personal care, lifting, reading, headaches, concentration, work, driving, sleeping, and recreation.

Scoring system: consists of 10 items, each with a score (0 to 5), for a total score of 50. The lower the score, the less self-rated disability. Grading of neck pain and disability index questionnaire was as follows: No disability (04), Mild disability (5-14), Moderate disability (15-24), severe disability (25-34), and Complete disability equals 35 or over.

Tool three: Visual analogue scale for pain (VAS pain): The VAS pain adopted from Katz \& Melzack (1999) consists of a $100 \mathrm{~mm}$ horizontal line with the words "no pain" and "worst pain imaginable" at the line's ends. Patients will be observed to quantify their neck pain by drawing a vertical mark on the area of 


\section{Thyroidectomy}

the horizontal line that best represented their pain level. Higher score $=$ more intense pain .

Scoring system of VAS includes: no pain (0$4 \mathrm{~mm})$, mild pain $(5-44 \mathrm{~mm})$, moderate pain (45$74 \mathrm{~mm})$, and severe pain (75-100mm).

\section{Exercises program:}

It was designed by the researcher according to the actual and potential needs assessment of the study patients after reviewing the related literature consisted of three sessions divided into two parts:

$>$ Theoretical part included three sessions.

$>$ Practical part included two sessions.

\section{Validity and reliability:}

* The revision of the tools was done by a panel of five medical surgical nursing expertise to test the relevance and clarity of contents and minor modifications were done in the form of rephrasing and organization of some questions.

- Testing reliability of proposed tools was done by Alpha Cronbach's alpha test. It was used to test whether the questionnaire had internal consistency. Knowledge regarding neckstretching exercises after surgery, Knowledge regarding thyroid gland and thyroidectomy and total knowledge respectively tests) the reliability score of tool as above is $(0.745$, 0.869 and 0.807$)$ and validity score of tool is $(0.775,0.904$ and 0.840$)$ this indicated high total internal consistency of the used tool.

\section{Pilot study:}

It was done on $10 \%$ of both studied groups (3 in intervention group) and (3 in control group) assess the applicability of the study tools and estimate the proper time required for answering the required data. All participants in the pilot study were excluded from the main study.

\section{Field work:-}

\section{A-Assessment phase:-}

Assessment of patients' knowledge about total thyroidectomy, complications of total thyroidectomy, stretching exercises was done. This assessment shed light on current knowledge and practice about neck stretching exercises to detect the defect and help in developing the exercise program according to results.

\section{B-Planning phase:}

The researcher made exploratory visit to study setting to put plan for carrying out the study. Exercises program developed by the researcher according to patients' needs, moreover teaching materials such as

(Discussion, demonstration and booklet) prepared to help in covering theoretical and practical information.

\section{C-Implementation phase:}

1. Data collection was started and completed within 12 months (From beginning of September 2019 up to the end of August 2020).

2. The study was done during morning \& Afternoon shifts three times weekly during the time of the study (12 months).

$>$ First 6 months data collection and implementation of exercises program for intervention group.

$>$ Second 6 months of data collection, evaluation for control group .

3. Interview with available patients to explain the aim of the study, the effect of this study on their neck pain\& neck disability level after total thyroidectomy and took their approval to participate in the study prior to data collection. 4. The exercises program and demonstration and re-demonstration of exercises and booklet had received to the intervention group. While the control group had received the routine nursing care and data collected from them in pre, $1^{\text {st }}$ week, and 4 th week post- operative. 
5. The researcher collected data about knowledge regarding neck-stretching exercises, knowledge regarding thyroid gland, and thyroidectomy by using patient structured questionnaire before developing the exercise program.

6. The researcher developed the exercises program and booklet regarding knowledge about neck-stretching exercises, thyroid gland, and thyroidectomy according to their needs in simple Arabic language.

7. The exercises program\& booklet were given by the researcher to intervention group and were asked to apply its contents.

8. Post-test was done after the exercise program implementation in $1^{\text {st }}$ and $4^{\text {th }}$ week postoperative for both groups (intervention and control).

\section{Exercise program}

It was conducted by the researcher according to the actual needs' assessment of the study patients after reviewing the related literature, it was adapted from Lynn (2011); Monahan (2012); LeMone et al. (2015); Ayhan et al. (2016) and consisted of two parts:

1-Theoretical part: - Knowledge booklet given to each intervention group included three sessions.

2-Practical part: - Demonstration and redemonstration about neck stretching exercises was done for each patient in intervention group, it included two sessions.

\section{D-Evaluation phase:}

This phase used to evaluate the effect of exercise program on their knowledge, neck pain and disability for both studied groups (intervention and control) groups with total thyroidectomy by using the second tool (NDI), third tool (VAS) and they were carried out in the outpatient surgery clinics at Benha university hospital during the pre-specified time with each patient (after the 1st and 4th week) following the thyroidectomy surgery and this mainly evaluated the effect of applying the neck stretching exercise on the incidence of neck pain and disability following total thyroidectomy.

\section{Statistical Analysis:}

Recorded data were analyzed using the statistical package for social sciences, version 20.0 (SPSS Inc., Chicago, Illinois, USA). Quantitative data were expressed as mean \pm standard deviation (SD). Qualitative data were expressed as frequency and percentage.

The following tests were done:

- Chi-square (x2) test of significance was used in order to compare proportions between qualitative parameters.

- Independent-samples t-test of significance was used when comparing between two means.

- A one-way analysis of variance (ANOVA) when comparing between more than two means.

- Linear regression: It is used to test and estimate the dependence of a quantitative variable based on its relationship to one or more independent variables.

- The confidence interval was set to $95 \%$ and the margin of error accepted was set to $5 \%$. So, the p-value was considered significant as the following:

- Probability (P-value) -P-value <0.05 was considered significant.

\section{Results:-}

Table (1): Showed that there was no significant statistical difference between both studied (intervention\& control) groups, regarding their socio-demographic data their mean age was(39.20 $\pm 7.45 \quad \& 40.51 \pm 7.70$, respectively),also nearly more than three quarters $(73.3 \& 80.0$ respectively) were female and also(83.3\&80.0, respectively) were married and regarding their level of education (33.3\&46.7, respectively) were highly educated and(83.3\&80.0,respectively) were working .Moreover, around two thirds of them (70.0\&66.7,respectively) were residing in rural areas. 


\section{Thyroidectomy}

Table (2): Revealed that, there was no significant statistical difference between both studied (intervention \& control) groups regarding their current medical history, concerning period of period from diagnosis of disease till the operation, the mean period was $(8.80 \pm 2.739 \& 80 \pm 3.04$, respectively) and according to cause of total thyroidectomy about one third (33.3\&36.7, respectively) had undergone thyroidectomy due to cancer of the thyroid gland, besides their main complains after surgery as neck pain as experienced by (26.7\&30.0, respectively) of both studied groups.

Table (3): Revealed that there was no significant statistical difference between both studied (intervention \& control) groups regarding their past medical history where (50.0 \&60.0, respectively) had no previous surgery, with (50.0\&63.3, respectively), had no chronic illness, while (50.0\&36.7,respectively) had chronic diseases, especially DM among(53.3\&63.6, respectively) of them, also around two thirds of them (66.7) for both groups had no family history for thyroidectomy and on the positive one third of them had mainly undergone total or sub -total thyroidectomy.

Table (4): Showed that comparison between both studied (intervention and control) groups regarding their knowledge level during (Preoperative, $1^{\text {st }}$ week postoperative, $4^{\text {th }}$ week postoperative) periods and clarified that there was no significant statistical differences between them regarding their total knowledge level about thyroid gland and thyroidectomy \& neck stretching exercises during pre-operative period. However, it was significantly different during (post-operative $1^{\text {st }}$ week $\& 4^{\text {th }}$ week), where the majority $(90 \%)$ of study group had satisfactory level of total knowledge, while the minority (10.0\%\&6.7\%, respectively) of control group had satisfactory level of total knowledge.

Figure (1): Showed that both studied (intervention and control) groups had unsatisfactory level of total knowledge at preoperative period, while the intervention group had higher satisfactory level of total knowledge at (1st week postoperative, 4th week postoperative) periods than the control group

Table (5): Revealed that there was highly statistically significant difference between both studied groups regarding their neck disability level in 1st week postoperative period, while there were statistically significant differences between both studied groups regarding their neck disability level in 4th week postoperative period.

Table (6): Revealed that there was highly statistically significant difference between both studied groups regarding their neck pain level in 1 st week postoperative period, while there were statistically significant differences between groups regarding their neck pain level in 4 th week postoperative period . 
Table (1): Distribution of studied patients group according to their socio-demographic data, both intervention group $(n=30)$ and control group $(n=30)$.

\begin{tabular}{|c|c|c|c|c|c|c|}
\hline \multirow[t]{2}{*}{ Socio-Demographic data } & \multicolumn{2}{|c|}{$\begin{array}{l}\text { Intervention } \\
\text { group }(\mathbf{n}=\mathbf{3 0})\end{array}$} & \multicolumn{2}{|c|}{ Control group $(n=30)$} & \multicolumn{2}{|c|}{ Chi-square test } \\
\hline & No. & $\%$ & No. & $\%$ & $\mathbf{x}^{2}$ & p-value \\
\hline \multicolumn{7}{|l|}{ Age (years) } \\
\hline $25<35$ years & 15 & 50.0 & 11 & 36.7 & \multirow{5}{*}{2.726} & \multirow{5}{*}{0.605 (n.s) } \\
\hline $35<45$ years & 6 & 20.0 & 10 & 33.3 & & \\
\hline $45<55$ years & 5 & 16.7 & 4 & 13.3 & & \\
\hline $55<65$ years & 2 & 6.7 & 4 & 13.3 & & \\
\hline$\geq 65$ years & 2 & 6.7 & 1 & 3.3 & & \\
\hline Mean \pm SD & \multirow{2}{*}{\multicolumn{2}{|c|}{$\begin{array}{c}39.20 \pm 7.45 \\
25-67\end{array}$}} & \multicolumn{2}{|c|}{$40.51 \pm 7.70$} & & \\
\hline Range & & & & & $t=0.962$ & 0.418 (n.s) \\
\hline \multicolumn{7}{|l|}{ Gender } \\
\hline Males & 8 & 26.7 & 6 & 20.0 & \multirow{2}{*}{0.373} & \multirow{2}{*}{0.542 (n.s) } \\
\hline Females & 22 & 73.3 & 24 & 80.0 & & \\
\hline \multicolumn{7}{|l|}{ Marital Status } \\
\hline Single & 3 & 10.0 & 2 & 6.7 & \multirow{4}{*}{1.220} & \\
\hline Married & 25 & 83.3 & 24 & 80.0 & & \multirow{3}{*}{0.748 (n.s) } \\
\hline Divorced & 1 & 3.3 & 3 & 10.0 & & \\
\hline Widowed & 1 & 3.3 & 1 & 3.3 & & \\
\hline \multicolumn{7}{|l|}{ Level of education } \\
\hline Illiterate & 10 & 33.3 & 6 & 20.0 & & \multirow{4}{*}{0.596 (n.s) } \\
\hline Reads and writes & 4 & 13.3 & 3 & 10.0 & \multirow{3}{*}{1.886} & \\
\hline Intermediate & 6 & 20.0 & 7 & 23.3 & & \\
\hline High & 10 & 33.3 & 14 & 46.7 & & \\
\hline \multicolumn{7}{|l|}{ Occupation } \\
\hline Working & 25 & 83.3 & 24 & 80.0 & \multirow{2}{*}{0.111} & \multirow{2}{*}{0.739 (n.s) } \\
\hline Not working & 5 & 16.7 & 6 & 20.0 & & \\
\hline \multicolumn{7}{|l|}{ Residence } \\
\hline Rural & 21 & 70.0 & 20 & 66.7 & \multirow{2}{*}{0.077} & \multirow{2}{*}{0.781 (n.s) } \\
\hline Urban & 9 & 30.0 & 10 & 33.3 & & \\
\hline
\end{tabular}

(Note): not statistically significant at $p$-value $>0.05$. 


\section{Thyroidectomy}

Table (2):- Distribution of studied patients group according to their current medical history, both intervention group $(n=30)$ and control group $(n=30)$.

\begin{tabular}{|c|c|c|c|c|c|c|}
\hline \multirow[t]{2}{*}{ Current medical history } & \multicolumn{2}{|c|}{$\begin{array}{c}\text { Interventio } \\
\text { n group } \\
(\mathbf{n}=\mathbf{3 0})\end{array}$} & \multicolumn{2}{|c|}{$\begin{array}{l}\text { Control group } \\
\quad(\mathbf{n}=\mathbf{3 0})\end{array}$} & \multicolumn{2}{|c|}{$\begin{array}{c}\text { Chi-square } \\
\text { test }\end{array}$} \\
\hline & $\begin{array}{c}\text { No } \\
\text {. }\end{array}$ & $\%$ & No. & $\%$ & $x^{2}$ & $\begin{array}{c}\text { p- } \\
\text { value }\end{array}$ \\
\hline \multicolumn{7}{|l|}{$\begin{array}{l}\text { Period from diagnosis of disease till the } \\
\text { operation }\end{array}$} \\
\hline$<2$ months & 6 & 20.0 & 6 & 20.0 & & \\
\hline $2-<4$ months & 4 & 13.3 & 4 & 13.3 & & \\
\hline $4-<6$ months & 8 & 26.7 & 5 & 16.7 & 0.777 & 0.942 \\
\hline $6 m-<1$ year & 5 & 16.7 & 8 & 26.7 & & \\
\hline$>1$ year & 7 & 23.3 & 7 & 23.3 & & \\
\hline Mean \pm SD & \multicolumn{2}{|c|}{$8.80 \pm 2.73$} & \multicolumn{2}{|c|}{$9.80 \pm 3.04$} & $\mathrm{t}=1.341$ & $\begin{array}{l}0.185 \\
\text { (n.s) }\end{array}$ \\
\hline Causes of total thyroidectomy & & & & & & \\
\hline Hyper thyroidisim & 6 & 20.0 & 5 & 16.7 & & \\
\hline Benign tumors of the thyroid gland & 8 & 26.7 & 8 & 26.7 & & \\
\hline Cancer of the thyroid gland & 10 & 33.3 & 11 & 36.7 & 0.139 & 0.998 \\
\hline Chronic goiter & 3 & 10.0 & 3 & 10.0 & & \\
\hline Nodular goiter & 3 & 10.0 & 3 & 10.0 & & \\
\hline Complains after surgery & & & & & & \\
\hline Neck pain & 8 & 26.7 & 9 & 30.0 & & \\
\hline Difficulty in movement of the neck & 6 & 20.0 & 6 & 20.0 & & \\
\hline Hoarseness or weakness of voice & 3 & 10.0 & 4 & 13.3 & & \\
\hline Difficulty swallowing & 2 & 6.7 & 2 & 6.7 & 0868 & 0.997 \\
\hline Difficulty chewing & 2 & 6.7 & 2 & 6.7 & 0.868 & (n.s) \\
\hline Difficulty performing any activity & 3 & 10.0 & 3 & 10.0 & & \\
\hline Difficulty speaking & 2 & 6.7 & 2 & 6.7 & & \\
\hline Shoulder pain & 4 & 13.3 & 2 & 6.7 & & \\
\hline
\end{tabular}

(Note): not statistically significant at $\mathbf{p}$-value $>0.05$. 
Table (3): Distribution of studied patients group according to their past medical history, both study group $(n=30)$ and control group $(n=30)$.

\begin{tabular}{|c|c|c|c|c|c|c|}
\hline \multirow[t]{2}{*}{ Past medical history } & \multicolumn{2}{|c|}{$\begin{array}{l}\text { Intervention } \\
\text { group }(n=30)\end{array}$} & \multicolumn{2}{|c|}{ Control group $(n=30)$} & \multicolumn{2}{|c|}{ Chi-square test } \\
\hline & No. & $\%$ & No. & $\%$ & $\mathbf{x}^{2}$ & p-value \\
\hline Previous surgery & & & & & \multirow{7}{*}{2.313} & \multirow{7}{*}{$\begin{array}{l}0.315 \\
\text { (n.s) }\end{array}$} \\
\hline No & 15 & 50.0 & 18 & 60.0 & & \\
\hline Subtotal thyroidectomy & 13 & 43.3 & 12 & 40.0 & & \\
\hline Previous neck or head surgery & 2 & 6.7 & 0 & 0.0 & & \\
\hline Type of neck or head surgery & $\mathbf{n}=\mathbf{2}$ & & $\mathbf{n}=\mathbf{0}$ & & & \\
\hline Lymph node removal & 1 & 50.0 & 0 & 0.0 & & \\
\hline Plastic surgery & 1 & 50.0 & 0 & 0.0 & & \\
\hline \multicolumn{7}{|l|}{ Chronic diseases } \\
\hline No & 15 & 50.0 & 19 & 63.3 & \multirow{2}{*}{1.086} & \multirow{2}{*}{$\begin{array}{l}0.297 \\
\text { (n.s) }\end{array}$} \\
\hline Yes & 15 & 50.0 & 11 & 36.7 & & \\
\hline Type of disease: & $\mathrm{n}=15$ & & $\mathrm{n}=\mathbf{1 1}$ & & \multirow{8}{*}{3.537} & \multirow{8}{*}{$\begin{array}{l}0.316 \\
\text { (n.s) }\end{array}$} \\
\hline Diabetes & 8 & 53.3 & 7 & 63.6 & & \\
\hline Heart diseases & 0 & 0.0 & 0 & 0.0 & & \\
\hline High blood pressure & 4 & 26.7 & 4 & 36.4 & & \\
\hline Kidney diseases & 3 & 20.0 & 0 & 0.0 & & \\
\hline Respiratory diseases & 0 & 0.0 & 0 & 0.0 & & \\
\hline Malignant tumors & 0 & 0.0 & 0 & 0.0 & & \\
\hline Others & 0 & 0.0 & 0 & 0.0 & & \\
\hline \multicolumn{7}{|l|}{ Past family history for thyroidectomy } \\
\hline No & 20 & 66.7 & 20 & 66.7 & \multirow{2}{*}{0.000} & \multirow{2}{*}{$\begin{array}{l}1.000 \\
\text { (n.s) }\end{array}$} \\
\hline Yes & 10 & 33.3 & 10 & 33.3 & & \\
\hline Type of surgery $(n=10)$ & $\mathbf{n}=\mathbf{1 0}$ & & $\mathbf{n}=\mathbf{1 0}$ & & \multirow{4}{*}{1.111} & \multirow{4}{*}{$\begin{array}{l}0.774 \\
\text { (n.s) }\end{array}$} \\
\hline Hemi Thyroidectomy & 1 & 10.0 & 0 & 0.0 & & \\
\hline Subtotal Thyroidectomy & 4 & 40.0 & 5 & 50.0 & & \\
\hline Total Thyroidectomy & 5 & 50.0 & 5 & 50.0 & & \\
\hline
\end{tabular}

(n.s): not statistically significant at p-value $>0.05$ 


\section{Effectiveness of Neck Stretching Exercises on Neck Pain and Disability for Patients with Total Thyroidectomy}

Table (4): Comparison of knowledge level regarding (Thyroid gland, thyroidectomy and neck stretching exercises) between both studied groups throughout different study periods (Pre-operative, 1st week postoperative, 4th week postoperative) intervention group $(\mathbf{n}=30)$ and control group $(n=30)$.

\begin{tabular}{|c|c|c|c|c|c|c|c|c|c|c|}
\hline \multirow{3}{*}{$\begin{array}{l}\text { Total } \\
\text { Knowledge } \\
\text { about thyroid } \\
\text { gland and } \\
\text { Thyroidectomy } \\
\text { \& neck } \\
\text { stretching } \\
\text { exercises }\end{array}$} & \multicolumn{4}{|c|}{ Intervention group $(n=30)$} & \multicolumn{4}{|c|}{ Control group $(n=30)$} & \multirow{2}{*}{\multicolumn{2}{|c|}{$\begin{array}{c}\text { Chi-square } \\
\text { test }\end{array}$}} \\
\hline & \multicolumn{2}{|c|}{$\begin{array}{c}\text { Unsatisfactory }<60 \% \\
\leq 60 \%\end{array}$} & \multicolumn{2}{|c|}{$\begin{array}{c}\text { Satisfactory } \geq 60 \% \\
>60 \%\end{array}$} & \multicolumn{2}{|c|}{$\begin{array}{c}\text { Unsatisfactory }<60 \% \\
\leq 60 \%\end{array}$} & \multicolumn{2}{|c|}{$\begin{array}{c}\text { Satisfactory } \geq 60 \% \\
>60 \%\end{array}$} & & \\
\hline & No. & $\%$ & No. & $\%$ & No. & $\%$ & No. & $\%$ & $\mathbf{x}^{2}$ & p-value \\
\hline Pre-Operative & 28 & 93.3 & 2 & 6.7 & 28 & 93.3 & 2 & 6.7 & 0.00 & $1.00(\mathrm{n} . \mathrm{s})$ \\
\hline $\begin{array}{l}\text { 1st week Post- } \\
\text { Operative }\end{array}$ & 3 & 10.0 & 27 & 90.0 & 27 & 90.0 & 3 & 10.0 & 38.40 & $<0.001 * *$ \\
\hline $\begin{array}{l}\text { 4th weeks Post- } \\
\text { Operative }\end{array}$ & 3 & 10.0 & 27 & 90.0 & 28 & 93.3 & 2 & 6.7 & 41.71 & $<0.001 * *$ \\
\hline
\end{tabular}

(**): Highly statistically significant at p-value $<0.001$, (n. s): not statistically significant at p-value $>0.05$ 


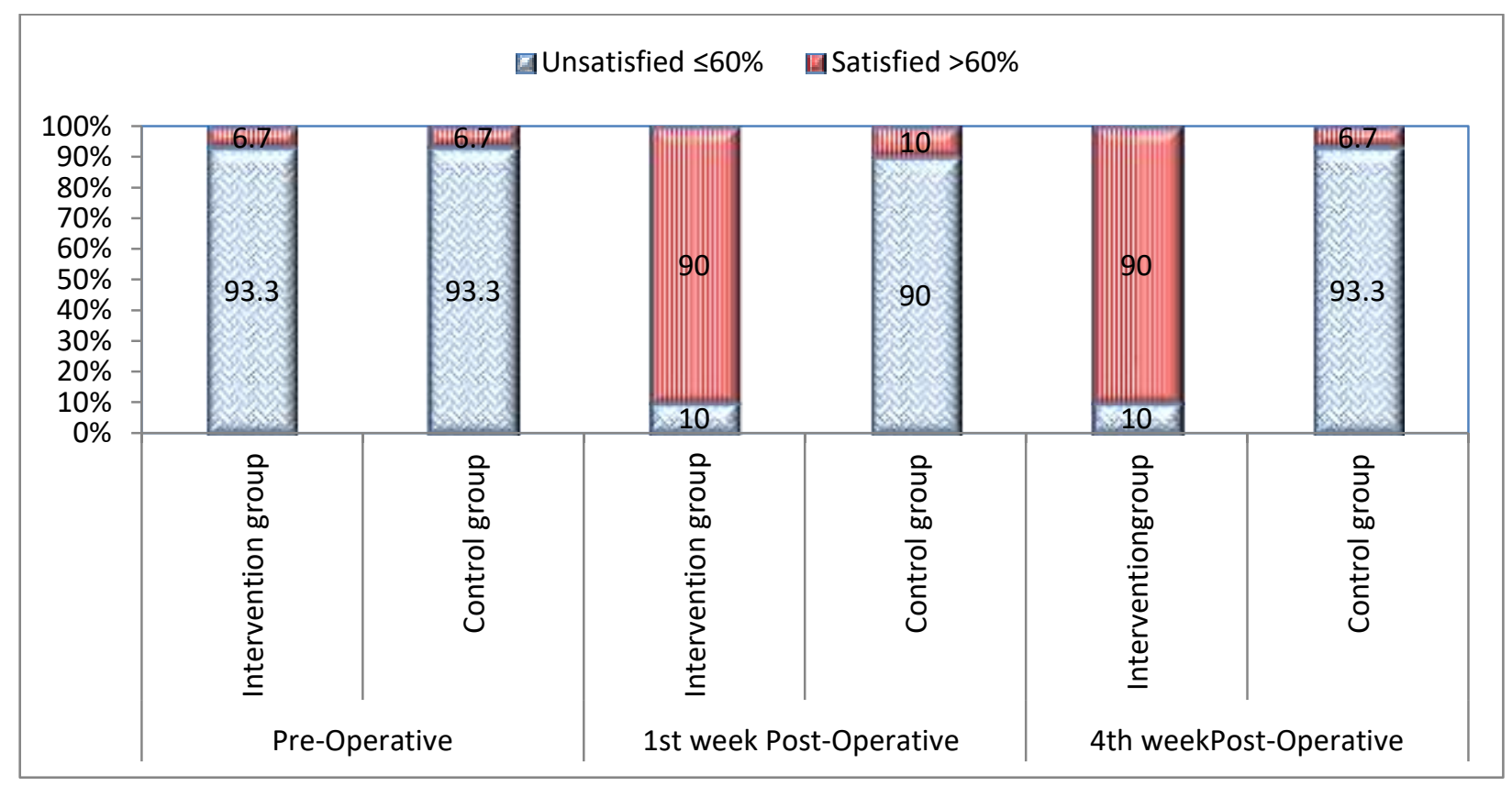

Figure (1):- Distribution of both studied (intervention and control) groups according to their total knowledge during (Pre-operative, $1^{\text {st }}$ week postoperative, $4^{\text {th }}$ week postoperative) periods.

Table (5):- Comparison between both studied groups regarding their neck disability level throughout different study periods $\left(1^{\text {st }}\right.$ week postoperative, $4^{\text {th }}$ week postoperative $)$ after program implementation intervention group $(n=30)$ and control group $(\mathbf{n}=\mathbf{3 0})$.

\begin{tabular}{|c|c|c|c|c|c|c|c|c|c|c|c|c|}
\hline \multirow{3}{*}{$\begin{array}{c}\text { Neck } \\
\text { disability } \\
\text { level }\end{array}$} & \multicolumn{6}{|c|}{ Post-Operative 1st week } & \multicolumn{6}{|c|}{ Post-Operative 4th weeks } \\
\hline & \multicolumn{2}{|c|}{$\begin{array}{c}\text { Intervention } \\
\text { group } \\
(\mathbf{n}=30)\end{array}$} & \multicolumn{2}{|c|}{$\begin{array}{c}\text { Control } \\
(n=30)\end{array}$} & \multirow[t]{2}{*}{$\mathbf{x} 2$} & \multirow[t]{2}{*}{ p-value } & \multicolumn{2}{|c|}{$\begin{array}{l}\text { Intervention } \\
\text { group } \\
(\mathbf{n}=30)\end{array}$} & \multicolumn{2}{|c|}{$\begin{array}{l}\text { Control } \\
(n=30)\end{array}$} & \multirow[t]{2}{*}{$\mathbf{x} 2$} & \multirow[t]{2}{*}{$\begin{array}{c}\text { p- } \\
\text { value }\end{array}$} \\
\hline & No. & $\%$ & No. & $\%$ & & & No. & $\%$ & No. & $\%$ & & \\
\hline $\begin{array}{l}\text { No } \\
\text { Disability }\end{array}$ & 16 & 53.3 & 3 & 10.0 & \multirow{5}{*}{24.367} & \multirow{5}{*}{$<0.001 * *$} & 29 & 96.7 & 21 & 70.0 & \multirow{5}{*}{7.947} & \multirow{5}{*}{$0.019 *$} \\
\hline $\begin{array}{l}\text { Mild } \\
\text { Disability }\end{array}$ & 8 & 26.7 & 3 & 10.0 & & & 1 & 3.3 & 5 & 16.7 & & \\
\hline $\begin{array}{l}\text { Moderate } \\
\text { Disability }\end{array}$ & 6 & 20.0 & 14 & 46.7 & & & 0 & 0.0 & 4 & 13.3 & & \\
\hline $\begin{array}{l}\text { Severe } \\
\text { Disability }\end{array}$ & 0 & 0.0 & 10 & 33.3 & & & 0 & 0.0 & 0 & 0.0 & & \\
\hline $\begin{array}{l}\text { Complete } \\
\text { Disability }\end{array}$ & 0 & 0.0 & 0 & 0.0 & & & 0 & 0.0 & 0 & 0.0 & & \\
\hline
\end{tabular}

$(* *)$ : Highly statistically significant at p-value $<0.001,\left(^{*}\right)$ : statistically significant at p-value $<0.05$ 


\section{Thyroidectomy}

Table (6):- Comparison between both studied groups regarding their neck pain level throughout different study periods $\left(1^{\text {st }}\right.$ week postoperative, $4^{\text {th }}$ week postoperative) after program implementation intervention group $(n=30)$ and control group $(\mathbf{n}=30)$.

\begin{tabular}{|c|c|c|c|c|c|c|c|c|c|c|c|c|}
\hline \multirow{3}{*}{ Neck pain level } & \multicolumn{6}{|c|}{ Post-Operative 1st week } & \multicolumn{6}{|c|}{ Post-Operative 4th weeks } \\
\hline & \multicolumn{2}{|c|}{$\begin{array}{l}\text { Study } \\
\text { group } \\
(n=30)\end{array}$} & \multicolumn{2}{|c|}{$\begin{array}{c}\text { Control } \\
(\mathbf{n}=\mathbf{3 0})\end{array}$} & \multirow[t]{2}{*}{$\mathbf{x} 2$} & \multirow[t]{2}{*}{ p-value } & \multicolumn{2}{|c|}{$\begin{array}{l}\text { Study } \\
\text { group } \\
(\mathrm{n}=30)\end{array}$} & \multicolumn{2}{|c|}{$\begin{array}{c}\text { Control } \\
(n=30)\end{array}$} & \multirow[t]{2}{*}{$\mathbf{x} 2$} & \multirow[t]{2}{*}{$\begin{array}{c}\text { p- } \\
\text { value }\end{array}$} \\
\hline & No. & $\%$ & No. & $\%$ & & & No. & $\%$ & No. & $\%$ & & \\
\hline No Pain & 15 & 50.0 & 2 & 6.7 & \multirow{4}{*}{28.974} & \multirow{4}{*}{$<0.001 * *$} & 30 & 100.0 & 25 & 83.3 & \multirow{4}{*}{5.455} & \multirow{4}{*}{$0.020 *$} \\
\hline Mild Pain & 10 & 33.3 & 3 & 10.0 & & & 0 & 0.0 & 5 & 16.7 & & \\
\hline Moderate Pain & 5 & 16.7 & 14 & 46.7 & & & 0 & 0.0 & 0 & 0.0 & & \\
\hline Severe Pain & 0 & 0.0 & 11 & 36.7 & & & 0 & 0.0 & 0 & 0.0 & & \\
\hline
\end{tabular}

$(* *)$ : Highly statistically significant at p-value $<0.001,(*)$ : statistically significant at p-value $<0.05$

\section{Discussion}

Diseases of thyroid gland are of great importance because they are a challenge for medical or surgical management. Total thyroidectomy is considered as the usual surgical procedure to treat thyroid diseases. The principal diseases of the thyroid gland are goiter, hypothyroidism, hyperthyroidism, thyroiditis, and neoplasms (Padur et al., 2016).

Complication rates are largely dependent on surgeon's skill and experience, the extent of surgery, and indication of surgery. Reported complications following thyroid surgery are rare but their consequences can often be life threatening as compared to other surgeries being performed routinely (Memon et al., 2012).

As regards to age of both studied groups, Regarding socio-demographic and medical data the current study showed that there was no significant differences between both groups reflecting the homogeneity of both groups regarding their characteristics, also as regards to age of both studied groups, the current study revealed that the mean age of both studied (intervention\& control) groups was 39.20 \pm 7.45
$\& 40.51 \pm 7.70$, respectively years old and nearly more than three quarters of them were females, these findings were supported by Abo Shehata et al., (2020) whose study entitled "Effect of neck range of motion exercises on neck disability and pain among patients undergoing Thyroidectomy" showed that the mean age of their both studied groups was $39.82 \pm 10.99 \&$ $38.93 \pm 10.88$ and about two-thirds of studied subjects were female for study and control groups respectively.

Regarding marital status, the current study revealed that more than more than three quarters of both studied (intervention\& control) groups were married. The study was congruent with Abd-El Mohsen and Ahmed (2018) whose study entitled "Effect of teaching patients neck stretching exercises on neck pain and disability following thyroidectomy" revealed that the majority of patients in the intervention and control groups were married.

As regards to the level of education, the current study revealed that more than one third of both studied groups were highly educated. The study was constituent with Nickel et al. (2019) whose study entitled 
"Health-Related Quality of Life after Diagnosis and Treatment of Differentiated Thyroid Cancer and Association with Type of Surgical Treatment" approved that most of the patients had post-secondary school level. Also these results agreed with Temiz et al. (2016) whose study entitled "Determination of Patient Learning Needs after Thyroidectomy" revealed that the studied patients who had a high school education level had higher mean scores.

Concerning patients' occupation, the majority of both studied groups were working. These results were in the same line with Abo Shehata et al. (2020) stated that more than half of the studied groups had worked. While these findings disagreed with Abd-El Mohsen and Ahmed (2018) showed that the majority of the studied groups were unemployed. Regarding their residence areas, around two thirds of them were residing in rural areas. These findings disagreed with Abo Shehata et al. (2020) stated that more than half of the studied groups were residing in urban areas.

Concerning current medical history, the period from diagnosis of disease till the operation, most of intervention group had less than 6 months period from diagnosis of disease till the operation but most of control group had more than 6 months period from diagnosis of disease till the operation, the study agreed with Nickel et al. (2019) stated that majority of studied patients had less than 6months period from diagnosis to treatment and minority of them had more than 6months period from diagnosis to treatment.

Regarding causes of total thyroidectomy, Regarding causes of total thyroidectomy, about one third of both studied groups had undergone thyroidectomy due to cancer of the thyroid gland. The study was congruent with Ayhan et al. (2016) whose study entitled "The Effectiveness of Neck Stretching Exercises
Following Total Thyroidectomy on Reducing Neck Pain and Disability: A Randomized Controlled Trial" stated that most of both studied groups had diagnosed with papillary thyroid carcinoma and had gone total thyroidectomy for this reason. Also this study was in the same line with Bhattacharyya and Fried (2002) whose study entitled with "Assessment of the morbidity and complications of total thyroidectomy" stated that the most common indication for surgery was thyroid malignancy about half of patients.

As regards to the complains after surgery, most of both studied groups had experienced neck pain. This may be due to the hyperextended position of neck during the operation. These findings supported by Takamura et al. (2005) whose study entitled "Stretching Exercises to Reduce Symptoms of Postoperative Neck Discomfort after Thyroid Surgery: Prospective Randomized Study" stated that both studied groups had complained about neck discomfort and pain after surgery. Also these findings congruent with Rodríguez et al. (2019) whose study entitled "Musculoskeletal neck disorders in thyroid cancer patients after thyroidectomy" stated that all patients had thyroidectomy complains of greater neck pain intensity and more disability.

Concerning past medical history, the current study revealed that half of intervention group and more than half of control group had no previous surgery, the results agreed with Abbas et al. (2019) whose study entitled "Challenges for huge goiter surgery" three patients who had previous surgery before but twenty-four patients had no surgery before.

\section{Related to presence of chronic diseases,} the present study showed that half of intervention group and more than half of control group had no chronic illness while more than half of them had chronic diseases, 


\section{Thyroidectomy}

especially DM. The study is in the same line with Turkmen (2020) whose study entitled "The Effect of Head-Neck Stretching Exercises after Thyroidectomy on Postoperative Pain Level and Wound Healing- Randomized Controlled Trial" stated that two thirds of both studied groups had no chronic illness while nearly half of them had chronic diseases but disagreed with this study that most of experimental group had heart failure and majority of control group had hypertension.

\section{Pertaining to the Past family history for} thyroidectomy, the current study revealed that around two thirds of both studied groups had no family history for thyroidectomy and third of them had positive family history mainly undergone total or sub -total thyroidectomy, the study in the same line with Attaallah et al. (2015) whose study entitled "Is hemithyroidectomy a rational management for benign nodular goitre? A Multicentre Retrospective Single Group Study" reported that most of the studied patients had had no family history for thyroid surgery but four patients only had a history of thyroid surgery.

Regarding comparing knowledge level about (Thyroid gland, thyroidectomy and neck stretching exercises) between both studied groups throughout different study periods (Pre-operative, 1st week postoperative, 4th week postoperative), the current study revealed that there was no significant statistical differences between them regarding their total knowledge level about thyroid gland and thyroidectomy \& neck stretching exercises during pre-operative period. However, it was significantly different during (post-operative 1st week \&4th week), where the majority of intervention group had satisfactory level of total knowledge, while the minority of control group had satisfactory level of total knowledge. This result supports the study hypothesis which suggested that the intervention group will exhibit higher knowledge level compared to control group after implementing the program. These findings constituent with Hassan et al., (2012) whose study entitled (Impact of a Designed Educational Program on Thyroidectomy Patients' Discharge Compliance Instructions) there was statistical significant difference between pre \&post-test of discharged knowledge regarding overall discharge compliance.

As regard to the neck disability level, the current study revealed that As regard to the neck disability level, the current study revealed that there was highly statistically significant difference between both studied groups regarding their neck disability level in 1st week postoperative period, while there were statistically significant differences between both studied groups regarding their neck disability level in 4th week postoperative period. This might be due to that neck disability ameliorates naturally within 1 month after thyroidectomy. This result supports the study hypothesis (2) which suggested that the intervention group will exhibit lower level of neck disability than control group after implementing the exercises program.

The study was congruent with Abd-El Mohsen\& Ahmed, (2018) ) showed that after one week of teaching the study group neck stretching exercises, more than half of them had no disability, about one third of patients were complaining from mild disability and only thirteen percent of them were complaining from moderate disability, no patient was complaining from severe or complete disability. On the other hand, more than one third of the control group subjects were complaining from severe disability and ten percent of them had no disability with 
statistically significant difference $(\mathrm{p}>.01)$. But the study results disagreed with him that after four weeks, the majority of patients, in both the study and control groups respectively, had no disability with no statistically significant difference.

Also the study agreed with Bhavani (2019) whose study entitled "Evaluating the Effects of Neck Exercise on Post Thyroidectomy PatientsA Pilot Study" stated that patients show a reduction in neck pain and disability after 1 week of exercise intervention.

As regard to the neck pain level, the current study revealed that there was highly statistically significant difference between both studied groups regarding their mean of neck pain level in 1st week postoperative period, while there were statistically significant differences between groups regarding their neck pain level in 4th week postoperative period. This might be due to that neck pain ameliorates naturally within 1 month after thyroidectomy. This result supports the study hypothesis (2) which suggested that the intervention group will exhibit lower level of neck pain than control group after implementing the exercises program.

This findings was in the same line with Ayhan et al. (2016) approved that it was found that patients experienced significantly less pain and disability in the stretching exercise group than the control group in post 1 week and post 1 month periods.

Also the study agreed with Abo Shehata et al. (2020) showed that there was a statistically significant difference between study and control group concerning neck disability and pain after one week and four weeks postoperative thyroidectomy .

As well as the current study agreed with Turkmen (2020) approved that there was a statistically significant difference between the mean scores of neck pain and disability 1 week after surgery and 1 month after surgery in the experimental and control groups $(\mathrm{p}<0.05)$.

\section{Conclusion}

There were high significant improvement in the intervention group total knowledge level during 1 st week and 4th week post - operative periods following the exercises program implementation more than the control group, moreover the intervention group had a lower level of neck pain and disability than the control group during 1st week and 4th week post - operative periods following the exercises program implementation.

\section{Recommendations:}

\section{Nursing recommendations:}

- Nurses working in the general surgery department must include the neck stretching exercises in care and followup protocols of patients undergoing total thyroidectomy.

- Standard nursing procedures booklet about total thyroidectomy and its complications and neck stretching exercises should be available at the surgical wards as a routine care in the pre-operative period to know how to deal with total thyroidectomy patients.

- Improving the quality of care given to patients in the out-patient clinics through continuous follow up for patient's neck disability and pain using validated tools in monitoring patient's condition.

\section{Researchers' recommendations:}

- Implementing educational programs for thyroidectomy patients about the thyroid disorders, medical and surgical management, complication of total 


\section{Thyroidectomy}

thyroidectomy and how to manage, preventing measures for early detecting and solving problems.

- Replication of the study using a larger probability sample from different geographical areas to attain more generalizable results.

\section{References}

Abbas, A., Sakkary, M., \& Naser, A. (2019). Challenges for huge goiter surgery. The Egyptian Journal of Surgery, 38(2), 338. http://www.ejs.eg.net/article.asp?issn=11101 121; year $=2019 ;$ volume $=38 ;$ issue $=2$; page $=3$ 38 ; epage $=347$; aulast $=$ Abbas

Abboud, B., Sleilaty, G., Rizk, H., Abadjian, G., \& Ghorra, C. (2012). Safety of thyroidectomy and cervical neck dissection without drains. Canadian Journal of Surgery, 55(3), 199.

https://www.ncbi.nlm.nih.gov/pmc/articles/p mc3364308/

Abd-El Mohsen, S. A., \& Ahmed, N. M. (2018). Effect of teaching patients neck stretching exercises on neck pain and disability following thyroidectomy. Journal of Nursing Education and Practice, 8(1).

https://www.academia.edu/download/55328 846/12077-42735-1-PB.pdf

\section{Abo Shehata,O., Shehata,O.\& Abd Elalem,S.} (2020). Effect of neck range of motion exercises on neck disability and pain among patients undergoing thyroidectomy, Clinical Nursing Studies,8(4).

http://www.sciedupress.com/journal/index.p hp/cns/article/view/18060

Al Shahrani, A. S., El-Metwally, A., Al-Surimi, K., Salih, S. B., Saleh, Y., Al-Shehri, A., \& Ali, A. (2016). The epidemiology of thyroid diseases in the Arab world: A systematic review. Journal of Public health and Epidemiology, 8(2), 17-26. https://pdfs.semanticscholar.org/3a6e/8d746 47bd42bdc312012f541e6da6bd55eba.pdf

Attaallah, W., Erel, S., Cantürk, N. Z., Erbil, Y., Görgülü, S., Kulaçoğlu, H., \& Güllüoğlu, B. M. (2015). Is hemithyroidectomy a rational management for benign nodular goitre? A Multicentre Retrospective Single Group Study. The Netherlands Journal of Medicine, 37(1). http://openaccess.maltepe.edu.tr/xmlui/handl e/20.500.12415/999

Ayhan, H., Tastan, S., Iyigün, E., Oztürk, E., Yildiz, R., \& Görgülü, S. (2016). The effectiveness of neck stretching exercises following total thyroidectomy on reducing neck pain and disability: a randomized controlled trial. Worldviews on Evidence-Based Nursing, 13(3), 224-231.

https://sigmapubs.onlinelibrary.wiley.com/d oi/abs/10.1111/wvn.12136

Bhattacharyya, N., \& Fried, M. P. (2002). Assessment of the morbidity and complications of total thyroidectomy. Archives of Otolaryngology-Head \& Neck Surgery, 128(4), 389-392.

https://jamanetwork.com/journals/jamaotola ryngology/article-abstract/482819

Bhavani, D. E., Monisha, R. and Kamalanathan, R. (2019). Evaluvating the Effects of Neck Exercise on Post Thyroidectomy Patients-A Pilot Study. Indian Journal of Public Health Research \& Development, 10(8):78-81.

https://wjpr.s3.ap-south

1.amazonaws.com/article_issue/1538202908 .pdf

Christou, N., \& Mathonnet, M. (2013). Complications after total thyroidectomy. Journal of visceral surgery, 150(4), 249-256.

https://www.sciencedirect.com/science/articl e/pii/S187878861300060X 
D'Orazi, V., Sacconi, A., Trombetta, S., Karpathiotakis, M., Pichelli, D., Di Lorenzo, E., \& Ortensi, A. (2019). May predictors of difficulty in thyroid surgery increase the incidence of complications?Prospective study with the proposal of a preoperative score. BMC surgery, 18(1), 1-8.

https://bmcsurg.biomedcentral.com/articles/ $10.1186 / \mathrm{s} 12893018-0447-7$

Hameed, A.T. (2018). Effectiveness of an Educational Program on Nurses' Knowledge and Practices about Prevent Complication for Patients Undergoing Thyroidectomy at AlHussein Teaching Hospital in Al-Nasiriyah City. Journal of Global Pharma Technology.

https://www.researchgate.net/profile/Mocham mad_Hatta/publication/327813841_A_revie w_Worldwide_medicinal_plants_for_typhoi d_fever/links/5ebf93ce299bf1c09ac0ba49/A -review-Worldwide-medicinal-plants-fortyphoidfever.pdf\#page $=87$

Hassan, A., El-Sayed, S., \& Taha, M. (2012). Impact of a Designed Educational Program on Thyroidectomy Patients' Discharge Compliance Instructions. Journal of American Science, 8(11), 1-13.

https://scholar.cu.edu.eg/sites/default/files/m edial_sector/files/032_11526am0811_237_2 49.pdf

Ignatavicius, D. D., \& Workman, M. L. (2015). Medical-Surgical Nursing-E-Book: PatientCentered Collaborative Care. Elsevier Health Sciences.

Jang, J. Y., Chang, Y. S., Kim, E. H., Moon, J. H., \& Son, Y. I. (2014). Early neck exercises to reduce post-thyroidectomy syndrome after uncomplicated thyroid surgery: a prospective randomized study. Journal of Korean Thyroid Association, 7(1), 70-76.

http://www.koreamed.org/SearchBasic.php? $\underline{\mathrm{RID}=2325294}$
Katz, J. \& Melzack, R. (1999). Measurement of pain. Surg Clin North Am; 79:231-52.

https://pubs.asahq.org/anesthesiology/article /95/6/1356/39229/The-Visual-Analog-Scalefor-PainClinical

LeMone, P., Burke, K., Dwyer, T., LevettJones, T., Moxham, L., \& Reid-Searl, K. (2015). Medical-surgical nursing. Pearson Higher Education AU,pp: 158-168.

Lewis, S. L., Bucher, L., Heitkemper, . M., Harding, M. M., Kwong, J., \& Roberts, D. (2016). Medical-Surgical Nursing-E-Book: Assessment and Management of Clinical Problems, Single Volume. Elsevier Health.

Lynn, P. (2011). Taylor's Clinical Nursing Skills: a nursing process approach (3rd ed.) , Philadelphia: Wolters Kluwer Health, 286-94, 366-71.

Memon, A. A., Junejo, A., \& balouch, T. A. (2012). Postoperative complications of thyroidectomy-An experience at teriatry care hospital. Medical Channel, 18(4).

http://search.ebscohost.com/login.aspx?direc $\mathrm{t}=$ true \&profile $=$ ehost \&scope=site\&authtype $=$ crawler \&jrnl $=16815491 \& \mathrm{AN}=100344918$ $\& \mathrm{~h}=\mathrm{iuMVdNmC9ZrdL38Tquge24TY2605If}$ ESwOSGxML7tC\%2B5QpSMru2KTrXZ6E gtb\%2FrwacYrMHZWz4ud4wzSQAKadA $\% 3 \mathrm{D} \% 3 \mathrm{D} \& \mathrm{crl}=\mathrm{c}$

Monahan, F. D. (2012). Phipps' medical-surgical nursing., Elsevier Mosby company, 222-35.

Nickel, B., Tan, T., Cvejic, E., Baade, P., McLeod, D. S., Pandeya, N., \& Jordan, S. (2019). Health-related quality of life after diagnosis and treatment of differentiated thyroid cancer and association with type of surgical treatment. JAMA otolaryngologyhead \& neck surgery, 145(3), 231-238. https://jamanetwork.com/journals/jamaotola ryngology/article-abstract/2721031 


\section{Thyroidectomy}

Padur, A. A., Kumar, N., Guru, A., Badagabettu, S. N., Shanthakumar, S. R., Virupakshamurthy, M. B., \& Patil, J. (2016): Safety and effectiveness of total thyroidectomy and its comparison with subtotal thyroidectomy and other thyroid surgeries: a systematic review. Journal of thyroid research. https://www.hindawi.com/journals/jtr/2016/ $7594615 / \mathrm{abs} /$

Rodríguez.Torres, J., López-López, L., Cabrera-Martos, I., Torres-Sánchez, I., Ortíz- Rubio, A., \& Valenza, M. C. (2019): Musculoskeletal neck disorders in thyroid cancer patients after thyroidectomy. European Journal of Cancer Care; 28(4): e13053.

https://onlinelibrary.wiley.com/doi/abs/10.1

111/ecc.13053

Statistical Administration and Medical Records, at Benha University Hospital, 2019.

Takamura, Y., Miyauchi, A., Tomoda, C., Uruno, T., Ito, Y., Miya, A., \& Kuma, K. (2005): Stretching exercises to reduce symptoms of postoperative neck discomfort after thyroid surgery: prospective randomized study. World journal of surgery, 29(6), 775779.

https://link.springer.com/article/10.1007/s00 268-005-7722-3

Temiz, Z., Ozturk, D., Ugras, G. A., Oztekin, S. D., \& Sengul, E. (2016): Determination of patient learning needs after thyroidectomy. Asian pacific journal of cancer prevention, 17(3), 1479-1483.

https://www.koreascience.or.kr/article/JAK

O201613752759290.page

Turkmen, A. (2020): The Effect of Head-Neck Stretching Exercises after Thyroidectomy on Postoperative Pain Level and Wound Healing .UN Published study in Cukurova University, Turkey, ClinicalTrials.gov, national library of medicine.
https://clinicaltrials.gov/ProvidedDocs/54/N CT04680754/Prot_SAP_000.pdf

Vernon,H., \&Mior, S., (1991): The neck disability index: A study of reliability and validity. Journal of Manipulative and Physiological Therapeutics, 14, 409-415. https://psycnet.apa.org/record/2018-23295001 


\section{تأثثر تمارين شد الرقبة على آلام وإعاقة الرقبة لمرضى الإستئصال الكلى للغدة الدرقية}

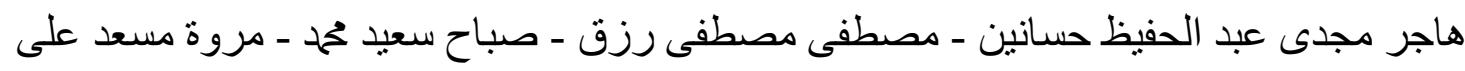

كثيرًا ما يعاني مرضى الإستئصال الكلى للغدة الدرقية من وجود ألم بالرقبة وضغط في الرقبة وتصلب الكتف وصعوبة الحركة في الرقبة و الكتف و تحدث هذه الأعر اض لأن وضع الرقبة يظل مددودا لفترة طويلة

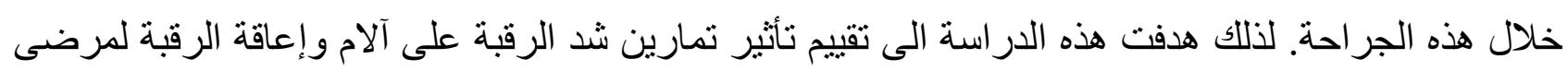

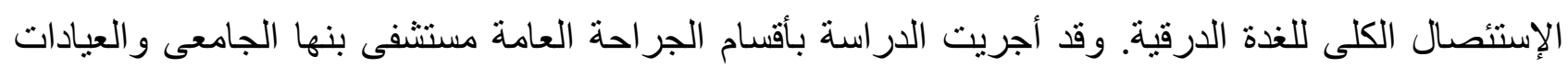

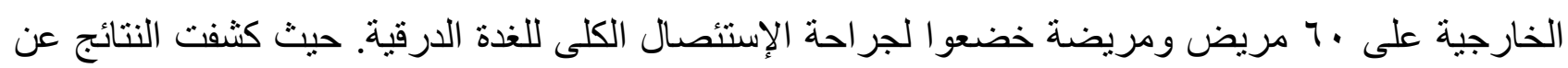

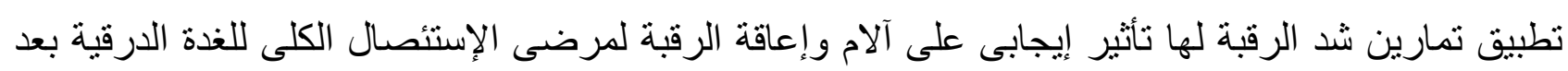

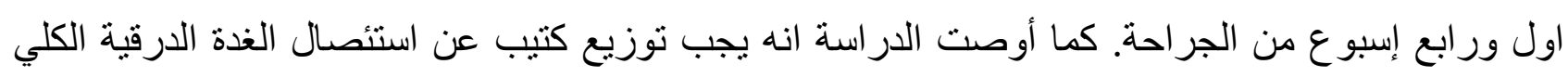

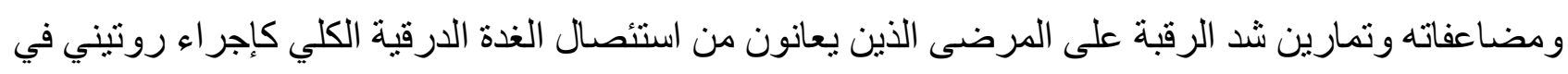
فترة ما قبل إجر اء الجراحة. 